\title{
White Eyelashes and Red Eyes in a 7-Year- Old Boy
}

Isabella Plumptre ${ }^{1} B A$, Satyamaanasa Polubothu ${ }^{2,3} M D$, Dhanes Thomas ${ }^{4} M D$, Veronica Kinsler ${ }^{2,3} M D P h D$

${ }^{1}$ Faculty of Medicine, Imperial College London, London, UK

${ }^{2}$ Genetics and Genomic Medicine, UCL Great Ormond St Institute of Child Health, London, WC1N 1EH, UK.

${ }^{3}$ Paediatric Dermatology, Great Ormond Street Hospital for Children NHS Foundation Trust, London WC1N 3JH, UK

${ }^{4}$ Moorfields Eye Hospital NHS Foundation Trust, 162 City Rd, London EC1V 2PD

Contact information for corresponding author: Veronica Kinsler, Paediatric Dermatology, Great Ormond Street Hospital for Children NHS Foundation Trust, London WC1N 3JH, UK email: v.kinsler@ucl.ac.uk; tel: 02074059200 ext 5601

Word count: 838/850 (Discussion 589/600)

Consent for publication from all authors: agreed

Conflict of interest declaration from all authors: no conflict of interest

Notice of informed consent from parents/guardians and/or patient: Informed consent obtained from parent 


\section{Case Presentation}

A 7 year old boy presented to Paediatric Dermatology with a history of white tinges within his eyelashes, followed two weeks later by hypopigmented patches and some areas of hair loss on the scalp, and an area of cutaneous hypopigmentation on the lower back.

Four months previously, the patient had experienced acute-onset painless bilateral red eyes and reduced visual acuity, which improved with steroid eye drops.

The patient had remained systemically well throughout, with no fevers, weight loss, sweats, fatigue, arthropathy, rash or neurological symptoms. There was no history of preceding illness or ocular trauma, no significant past medical history, and no family history of note.

On examination, there was patchy hypopigmentation within the eyelashes (figure 1) and scalp hair, areas of alopecia with some regrowth (figure 2), and roughly symmetrical bilateral patchy hypopigmentation of the lower lumbar area (figure 3). Blood tests, including a full blood count, antinuclear antibodies, HLA-B27 screen, quantiferon and toxoplasmosis serology, showed no abnormalities. An MRI of the brain was also normal.

\section{What is the diagnosis?}




\section{Clinical Course}

The combination of acute panuveitis, poliosis, alopecia and cutaneous hypopigmentation was sufficient in this case for a clinical diagnosis of Vogt-Koyanagi-Harada disease. Although topical (and later oral) steroids improved the patient's visual acuity, these were discontinued due to raised intraocular pressure. Oral methotrexate (15mg weekly) was started as an alternative, which resolved the eye disease and improved the poliosis and alopecia. The hypopigmented lumbar patches remain unchanged, 6 months after initial presentation.

\section{Discussion}

Vogt-Koyanagi-Harada disease (VKHD) is a rare systemic autoimmune condition targeting melanocytes in different tissues, including the eye, the brain, the inner ear and the skin (1). The presentation is variable according to which tissues are affected and the chronological course of the disease.

The diagnosis is made clinically and can be classified as complete, incomplete or probable VKHD as dictated by set criteria (2). There are no diagnostic serological or histological findings. VKHD is an important diagnosis to make as there is a significant threat of visual loss; acutely from retinal detachment, and/or chronically from glaucoma, cataracts and choroidal neovascularization. However, these serious and debilitating consequences are preventable with prompt diagnosis and treatment.

Differential diagnosis of the cutaneous signs theoretically includes vitiligo and causes of patchy alopecia, and it is therefore critical to ask for a history of uveitis to discriminate from 
these. Alezzandrini syndrome is a possibly separate, possibly overlapping differential diagnosis of VKHD (3).

There are four classic stages of VKHD. The first is a prodromal stage of non-specific flu-like illness. This is followed days later by the second and characteristic acute uveitic phase, which gives rise to several weeks of blurred vision and redness. This acute phase can also be associated with headache, tinnitus and neck stiffness. A few months later, the disease enters the third phase, that of chronic hypo- or de-pigmentation, which affects the eyes and skin. In the eyes, depigmentation of the choroid ultimately results in a 'sunset-glow fundus'. Dermatological findings include alopecia, poliosis of the scalp, eyebrows and eyelashes, and vitiligo. The lower back is the most classic area affected by hypo or de-pigmentation, as seen in this patient. Periumbilical depigmentation (Sugiura's sign) can also occur at an earlier stage, around a month after the onset of uveitis (4). The fourth stage is chronic recurrent disease, in which patients can experience flares of uveitis, ocular complications (glaucoma, cataracts and choroidal neovascularization) and ongoing dermatological involvement. VKHD predominantly affects those of Asian and Hispanic descent. However, this appears to be related to ethnic group rather than degree of skin pigmentation, as VKHD is rare in African-Americans (5). Although more common in adults (1), VKHD can present in childhood. Indeed, VKHD is responsible for an estimated $16 \%$ of pediatric uveitis in Saudi Arabia (6). Typically, the prognosis of VKHD in children is good (7). However, VKHD presenting under the age of 14 appears to result in more aggressive disease with poorer visual outcomes (8). Early initiation of high-dose steroid therapy is key in the treatment of VKHD. As evidence for treatment in children is limited, adult literature is used as the basis for treatment. Systemic prednisone, either oral or intravenous, rapidly improves acute symptoms by resolving 
exudative retinal detachment. In children, a shorter interval between symptoms and treatment is linked to better final outcomes of visual acuity (7). Oral steroid therapy should be gradually reduced over two years to prevent recurrence (9). Patients with steroidresistant disease or steroid intolerance can be treated with methotrexate or other antimetabolites as a second line therapy.

The mechanism underlying VKHD is not fully understood, but is currently considered to be an autoimmune process targeting unidentified melanocyte-associated antigens, preceded by viral infection (10).

In summary, VKHD is a serious autoimmune condition that presents with acute uveitis followed months later by poliosis, alopecia and vitiligo. Both acute and chronic disease can lead to permanent visual loss, which is preventable with early initiation of steroid therapy, and recognition of the disease by Paediatric Dermatologists could therefore be important in directing therapy.

\section{References}

1. Moorthy RS, Inomata H, Rao NA. MAJOR REVI EW Vogt-Koyanagi-Harada Syndrome. Surv Ophthalmol. 1995;39(4).

2. Read RW, Holland GN, Rao N a, Tabbara KF, Ohno S, Arellanes-Garcia L, et al. Revised diagnostic criteria for Vogt-Koyanagi-Harada disease: report of an international committee on nomenclature. Am J Ophthalmol. 2001;131(5):647-52.

3. Casala A, Alezzandrini A. Vitiligo, poliosis unilateral con retinitis pigmentaria y hypoacusia. Arch Argent Dermatol. 1959;(9):449. 
4. Sugiura S. [Some observations on uveitis in Japan, with special reference to VogtKoyanagi-Harada and Behcet diseases]. Nihon Ganka Gakkai Zasshi. Japan; 1976 Nov;80(11):1285-326.

5. Lin GG, Scott JG. Frequency of Distinguishing Clinical Features in VogtKoyanagiHarada disease. Opthalmology. 2010;117(3):591-9.

6. Hamade IH, Al Shamsi HN, Al Dhibi H, Chacra CB, Abu El-Asrar a M, Tabbara KF. Uveitis survey in children. Br J Ophthalmol. 2009;93:569-72.

7. Abu El-Asrar AM, Al-Kharashi AS, Aldibhi H, Al-Fraykh H, Kangave D. Vogt-KoyanagiHarada disease in children. Eye. 2008;22(9):1124-31.

8. Tabbara KF, Chavis PS, Freeman WR. Vogt-Koyanagi-Harada syndrome in children compared to adults. Acta Ophthalmol Scand. 1998;76(6):723-6.

9. Baltmr A, Lightman S, Tomkins-Netzer O. Vogt - Koyanagi - Harada syndrome current perspectives. Clin Opthalmology. 2016;10:2345-61.

10. Lavezzo MM, Sakata VM, Morita C, Rodriguez EEC, Abdallah SF, da Silva FTG, et al. Vogt-Koyanagi-Harada disease: review of a rare autoimmune disease targeting antigens of melanocytes. Orphanet J Rare Dis. Orphanet Journal of Rare Diseases; 2016;11:29. 